\title{
Expression of LDL-A module of relaxin receptor in prostate cancer cells inhibits tumorigenesis
}

\author{
SHU FENG ${ }^{1,2}$ and ALEXANDER I. AGOULNIK ${ }^{1,3}$ \\ Departments of ${ }^{1}$ Obstetrics and Gynecology, and ${ }^{2}$ Pathology and Immunology, Baylor College of \\ Medicine, One Baylor Plaza, Houston, TX 77030; ${ }^{3}$ Department of Human and Molecular Genetics, \\ Herbert Wertheim College of Medicine, Florida International University, Miami, FL 33199, USA
}

Received May 10, 2011; Accepted June 22, 2011

DOI: 10.3892/ijo.2011.1159

\begin{abstract}
Peptide hormone relaxin produced in normal and cancer prostate cells can stimulate prostate cancer progression. Suppression of relaxin or relaxin receptor RXFP1 expression inhibited prostate cancer both in vitro and in vivo. RXFP1 is a $G$ protein-coupled receptor with the extracellular low density lipoprotein A (LDL-A) module located at the N-terminus. The LDL-A module exhibits a competitive inhibition effect on the RXFP1 function and might be used to suppress relaxin signaling. In this study, we investigated the effect of LDL-A overexpression on prostate cancer cells. PC3 cells expressing the RXFP1-LDL-A module had a decreased proliferation, soft agar colony formation, adhesion, invasion in vitro, and grew at a slower rate than control cells as tumors in xenograft models in mice. Expression analysis revealed that the RXFP1LDL-A expression in PC3 cells inhibited Akt phosphorylation and MMP2 activation, and led to the down-regulation of several genes previously implicated in tumorigenesis, such as MMP28, S100P, IGFBP2, MUC1 and S100A4. These results indicate that the inhibition of RXFP1 by peptide based on the LDL-A module of this receptor may be a potential approach for prostate cancer suppression.
\end{abstract}

\section{Introduction}

Relaxin (RLN) is a small peptide hormone of relaxin/insulinlike family (1). Mouse relaxin shares only $48 \%$ identity at the amino acid level with human RLN2, but each is bioactive in both species. While relaxin role in female reproduction and specifically in pregnancy has been studied extensively, its functions in males are more controversial $(2,3)$. The expression of the hormone in males has been detected in prostate and

Correspondence to: Dr Alexander I. Agoulnik, Department of Human and Molecular Genetics, Herbert Wertheim College of Medicine, Florida International University, 11200 SW 8th Street, HLSI 419B, Miami, FL 33199, USA

E-mail: aagoulni@fiu.edu

Key words: relaxin receptor, LDL-A, prostate cancer in testis, but the concentration of relaxin in peripheral blood is significantly lower than that in females (3). On the other hand, the expression of relaxin receptor RXFP1, relaxin family peptide receptor 1 , is readily detectable in numerous organs (4). Gene targeting experiments in mice resulted in viable $R \ln 1$ and Rxfpl-deficient animals (5-7). Detailed analysis of their phenotype revealed an involvement of this peptide in extracellular matrix remodeling; a fibrosis development was described in various $R \ln 1$ - or $R x f p l$-deficient organs (8). The role of relaxin in regulation of angiogenesis and vasodilation was linked to the induction of vascular endothelial growth factor, up-regulation of nitric oxide synthase, matrix metalloproteases and other factors (9). Modulations of relaxin signaling affect cell proliferation, apoptosis, cell migration, invasiveness, adhesion and other cell characteristics important in the context of normal development as well as in oncogenic transformation. In the last several years the potential role of relaxin in prostate cancer tumorigenesis was under investigation (10-17). Relaxin and RXFP1 are well-expressed in normal epithelial prostate cells as well as in prostate cancer cells. We have shown that an increased relaxin expression in tumor patient samples correlated with aggressive metastatic disease and shorter overall survival (13). It was reported that the relaxin expression became up-regulated during prostate cancer progression to androgen independence and was negatively regulated by androgens (12). The experiments with androgen receptor positive and negative LNCaP and PC3 prostate adenocarcinoma cells revealed the stimulating effects of relaxin on cell proliferation, migration, and adhesion in vitro (13). Similarly, in the $\mathrm{LNCaP} / \mathrm{PC} 3$ xenograft experiments the overexpression of relaxin in cancer cells increased tumor growth, vascularization and metastasis rates $(11,16)$, whereas an overexpression of relaxin homolog with antagonistic properties decreased tumor growth (14). We have shown that systemic transgenic overexpression of relaxin in mice decreased survival of TRAMP (transgenic adenocarcinoma of mouse prostate) mutant males (13). Treatment of prostate cancer xenografts with chitosan nanoparticles containing siRNA targeting RXFP1 decreased tumor growth and number of metastases (17).

As other $\mathrm{G}$ protein-coupled receptors RXFP1 is expressed on the cell surface membrane and hence is an attractive target for therapeutic intervention. Stimulation of RXFP1 causes an activation of cAMP through Gs/Gi proteins and protein 
kinase A in some, but not all cells (18). An involvement of tyrosine kinases, protein kinase C zeta, PI3 kinase and other molecules in relaxin receptor signaling was also demonstrated (18). The extracellular part of the relaxin receptor contains 10 leucine-rich repeats and a low-density lipoprotein module A (LDL-A) at the N-terminus of the protein (4). The mutations in LDL-A abolish the receptor signaling, but not the hormone binding (18). Treatment of the RXFP1 transfected cells with a peptide designed based on LDL-A module suppressed relaxininduced cAMP activation, suggesting the antagonistic behavior (19). Taking into account the effect of relaxin signaling on tumor progression we analyzed the effect of RXFP1-LDL-A expression on cancer cells in vitro and in xenograft experiments in mice. The results indicated that the stable transfection of PC3 adenocarcinoma cells with the RXFP1-LDL-A expression vector suppressed tumorigenic properties of cancer cells with a corresponding decrease in the expression of several genes involved in prostate tumor progression.

\section{Material and methods}

Cell cultures. The PC3 cells, originally purchased from American Type Culture Collection, Inc. (Manassas, VA) were maintained in 1:1 mixture of Ham's Nutrient mixture F12 and DMEM (Invitrogen, Carlsbad, CA) supplemented with 10\% fetal bovine serum, 100 units penicillin/ml and $100 \mu \mathrm{g}$ streptomycin/ml (Invitrogen).

Production of RXFP1-LDL-A transfected PC3 cells. FLAGtagged human RXFP1-LDL-A mammalian expression construct FLAG-RXFP1-LDL-A/pcDNA3.1/Zeo was generated by PCR using human RXFP1-pcDNA3.1/Zeo plasmid (4) with a forward T7 primer and a reverse primer (5'-GGG GGC CCT TAC TCT CCA CAG TTG TCC TCA TC-3') containing an $A p a \mathrm{I}$ site. The resultant product was cloned into BamH1/ApaI sites of pcDNA3.1/Zeo and fully sequenced. The FLAG-RXFP1-LDL-A/pcDNA3.1/Zeo and vector pcDNA3.1/ Zeo plasmids were used to obtain stable transfected PC3 cell lines using the Nucleofector Kit V with Nucleofector device (Lonza Group Ltd., Gaithersburg, MD). Transfected cells were selected with $500 \mathrm{ng} / \mathrm{ml}$ Zeocin (Invitrogen, determined by the kill curve assay). Individual resistant clones were picked 3 weeks later and expanded into the cell lines.

Cell proliferation assay. Cell proliferation was estimated using the CellTiter 96 Aqueous One Solution Cell Proliferation Assay (Promega, Madison, WI) as previously described (13). Eight replicate per cell lines were assayed, and data averaged from three separate experiments are presented.

Adhesion assay. The adhesion of the stable transfected cells and the parental PC3 cells to type I collagen was determined as previously described (13). All measurements were repeated six times/group in three independent experiments.

Colony formation and soft agar colony formation assay. For colony formation assay, 5000 cells were plated in 6-well plates. The medium was changed every 3 days. On the 12 th day, the plates were stained with $1 \%$ crystal violet, and the colonies were quantified. Colony formation in soft agar was performed as previously described (13). Briefly, a suspension of 5000 cells $/ \mathrm{ml}$ cells was prepared in $0.35 \%$ agar diluted in growth medium and plated on a $3.5 \%$ agar foundation in 6-well culture plate. After 14 days culture, cells were stained with $2 \mathrm{mg} / \mathrm{ml}$ of p-iodonitrotetrazolium violet (Sigma, St. Louis, MO) at $37^{\circ} \mathrm{C}$ for $24 \mathrm{~h}$. Colonies were counted under dissecting microscope. Experiments were repeated three times.

Matrigel invasion assay. BD BioCoat Matrigel invasion Chambers with Matrigel inserts with $8 \mu \mathrm{m}$ pores (BD Biosciences, Bedford, MA) were used for cell invasion assay as previously described (13). All experiments were performed three times; each point was repeated in triplicate.

Western blot analysis. Specific RXFP1 and negative control siRNA were supplied by Qiagen (Valencia, CA). The siRNA sequence used for $R X F P l$ gene silencing (siRXFPl) was GGAUGUCAAGUGCUCCCUUtt (sense strand) $(13,17)$. Negative control siRNA (siNC) with no significant sequence similarity to human gene sequences was used as control. The PC3 cells were transfected with siRXFP1 and siNC (5 $\mu \mathrm{g}$ each) using Nucleofector device as previously described (17). Transfected cells were harvested after $72 \mathrm{~h}$, the protein was extracted from the total cell lysate for Western blot experiments. In parallel experiment, the protein was extracted from the FLAG-RXFP1-LDL-A and pcDNA3.1/Zeo vector control PC3 cells. The extracted proteins $(30 \mu \mathrm{g})$ were subjected to Western blot analysis using phospho-Akt (Thr308) or total Akt antibodies (1:1000, Cell Signaling Technology, Danvers, MA). A goat polyclonal anti- $\beta$-actin antibody (Santa Cruz Biotechnology, Santa Cruz, CA) at 1:1000 was used as an internal control.

Zymography. Gelatinolytic zymography was used to detect the activity of MMP-2 and MMP-9 in conditioned media of FLAG-RXFP1-LDL-A and vector control stable transfected PC3 cells. Briefly, cells were incubated in serum-free medium for $72 \mathrm{~h}$ in 6 -well plates. The conditioned media were collected by centrifuging at $4^{\circ} \mathrm{C}$ at $13200 \mathrm{rpm}$ for $30 \mathrm{~min}$. Samples of $20 \mu \mathrm{g}$ were mixed with non-denaturing loading buffer and separated in $10 \%$ SDS-polyacrylamide gels containing $1 \mathrm{mg} / \mathrm{ml}$ gelatin. After electrophoresis, gels were washed with $2.5 \%$ Triton $\mathrm{X}-100$ for $1 \mathrm{~h}$ three times to remove SDS before incubation for $24 \mathrm{~h}$ at $37^{\circ} \mathrm{C}$ in developing buffer $(50 \mathrm{mM}$ Tris- $\mathrm{HCl} \mathrm{pH} \mathrm{8.0,5} \mathrm{mM} \mathrm{CaCl}, 5 \mu \mathrm{M} \mathrm{ZnCl}_{2}$ and $0.02 \% \mathrm{NaN}_{3}$ ). Gels were fixed and stained with $50 \%$ methanol and $10 \%$ acetic acid containing $0.3 \% \mathrm{w} / \mathrm{v}$ Coomassie Blue. Experiments were performed in duplicate three times.

RNA isolation and RT-PCR. Total RNA was isolated from cells using the RNeasy kit (Qiagen, MD, USA) and digested with the DNA-free ${ }^{\mathrm{TM}}$ DNase Treatment and Removal Reagents (Ambion, Austin, TX), according to the manufacturer's protocol. cDNA was synthesized using an oligo(dT) primer and RETROscript Kit (Ambion). The FLAG-RXFP1-LDL-A expression was analyzed by RT-PCR with primers PRLsignalPep forward (5'-ATG GAC AGC AAA GGT TCG TC-3') and RXFP1LDL-A reverse (5'-TCT CCA CAG TTG TCC TCA TCG G-3'). RT-PCR with primers for $\beta$-actin gene, $\beta$-actin forward, 5'-CCAAGGCCAACCGCGAGAAGATGAC-3', and $\beta$-actin 
reverse, 5'-AGGGTACATGGTGGTGCCGCCAGAC-3', was used to assess the quality of cDNA pools. Real-time quantitative RT-PCR (QRT-PCR) was performed with a qPCR SYBR Green real-time protocol on the IQ5 iCycler (Bio-Rad, Hercules, CA). Primers for different genes are available upon the request. All data were normalized to GAPDH gene expression. Each point was done at least in triplicate and the average and standard errors were calculated.

Xenograft studies. All experiments were conducted using the standards for humane care in accordance with the NIH Guide for the Care and Use of Laboratory Animals and approved by the Baylor College of Medicine Institutional Committee. A total of $10^{7}$ RXFP1-LDL-A stable transfected PC3 cells (LDL5) or vector control cells (V14) in $200 \mu 1$ of a 1:1 mixture of DMEM/F12 and Matrigel (BD Biosciences, Bedford, MA) were injected subcutaneously into two abdominal flanks of 6-week male nude mice (12 per group) obtained from NCI Animal Production Program. The tumors were measured twice a week using a digital slide caliper and the tumor volume in cubic millimeters was calculated by the following formula: volume $=$ width $^{2} \mathrm{x}$ length $/ 2$. After eight weeks mice were sacrificed; tumors were dissected, lung, liver, kidneys, brain and lymph nodes were analyzed for the presence of metastases.

Immunohistochemistry. The tumor sections were prepared using standard protocol. Tumor cell proliferation was evaluated by immunohischemistry (IHC) using anti-Ki67 (1:100 dilution; Abcam, Cambridge, MA). Images were taken with a Nikon-TMS inverted microscope equipped with Olympus DP70 digital imaging system. Ki67 staining was scored by the percentage of positive tumor cells in eight random fields per section at $\mathrm{x} 400$ magnification in each tumor section.

Statistical analysis. Student's t-test was employed. All experiments were performed at least in triplicates and were expressed as mean \pm standard error, with $\mathrm{P}<0.05$ considered as statistically significant. Analysis of the xenograft growth was performed by two-way ANOVA using GraphPad Prism Software (La Jolla, CA).

\section{Results}

To determine the effect of LDL-A module of relaxin receptor overexpression on prostate cancer progression, we generated stable transfectants of PC3 cells that express RXFP1-LDL-A. PC3 cells were transfected with a human RXFP1-LDL-A mammalian expression construct FLAG-RXFP1-LDL-A/ pcDNA3.1zeo or pcDNA3.1zeo vector as a control (Fig. 1A). Presence of the plasmid DNA was confirmed by PCR with vector-specific primers (data not shown). Several individual clones were obtained for each group. The RXFP1-LDL-A mRNA expression was evaluated in 5 clones using RT-PCR with primers specific for PRL-SP and LDL-A as shown in Fig. 1B. The following clones from each group were used in further studies: control, V14; LDL-A, LDL5 or LDL8.

Effect of stable transfection of RXFPl-LDL-A on PC3 cell tumorigenic properties in vitro. To analyze the effect of
A

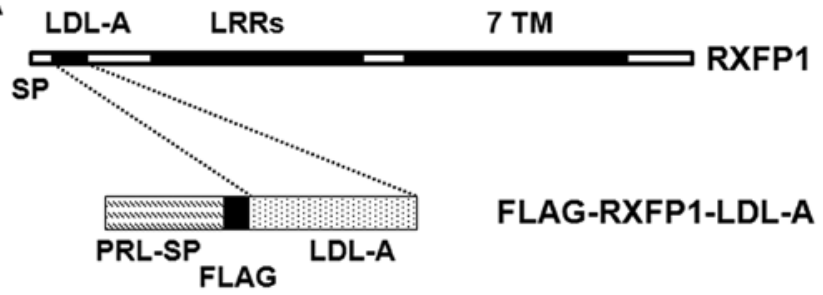

B

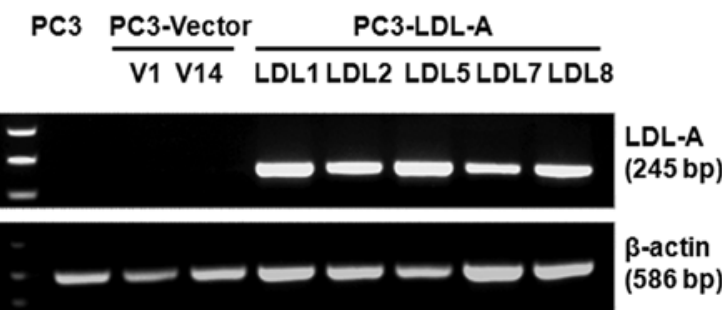

Figure 1. Generation of RXFP1-LDL-A PC3 stable transfectants expressing RXFP1-LDL-A peptide. (A) Structure of RXFP1-LDL-A expression vector. The RXFP1 protein contains signal peptide (SP), low density lipoprotein A module (LDL-A), leucine rich repeats (LRR) and seven transmembrane domains (7TM). The RXFP1-LDL-A expression vector contains DNA encoding bovine prolactin signal peptide (PRL-SP), FLAG epitope, and 128 bp of RXFP1-LDL-A module. (B) RT-PCR analysis of RXFP1-LDL-A (LDL) and vector (V) stably transfected cells for the expression of LDL-A domain.

RXFP1-LDL-A expression on cell growth, the parental PC3 cells, RXFP1-LDL-A stable transfectant LDL5 and LDL8 cell lines, and vector control V14 cells were used. Cell proliferation was determined at different time points. As shown in Fig. 2A, both LDL5 and LDL8 cells exhibited decreased proliferation compared to control V14 cells or parental PC3 cells. Adhesion assay showed that the LDL-A overexpression in LDL5 and LDL8 cells also inhibited cell adhesion to type I collagen (Fig. 2B). The transfected cells were also examined for colony formation and anchorage-independent growth in a soft agar medium. The results showed that LDL5 and LDL8 cells exhibited a lower colony formation capacity on solid surface of culture dishes compared to the vector control or parental PC3 cells (Fig. 3A). All cell lines exhibited anchorage-independent growth in soft agar. As shown in Fig. 3B, LDL5 and LDL8 cells formed fewer colonies in soft agar compared to the V14 and parental PC3 cells $(\mathrm{P}<0.001)$ suggesting an effect of RXFP1-LDL-A expression and a decreased survival/growth advantage in anchorage-independent condition. We tested whether RXFP1-LDL-A affected prostate cancer cell invasiveness in an in vitro Matrigel invasion assay. About two to three times fewer RXFP1-LDL-A expressing LDL5 and LDL8 cells traversed the Matrigel than did control V14 or PC3 cells (Fig. 4A). Gelatin zymography showed that the MMP2 activity in LDL5 cells was significantly lower compared to the control V14 cells (Fig. 4B), suggesting that the modulation of MMP2 activity might be responsible for the decreased invasiveness in LDL-A overexpressing cells.

Previously relaxin-induced modulation of Akt protein kinase B (PKB) phosphorylation was shown for LNCaP cells (15). The activity of Akt is implicated in mediating a variety 
A

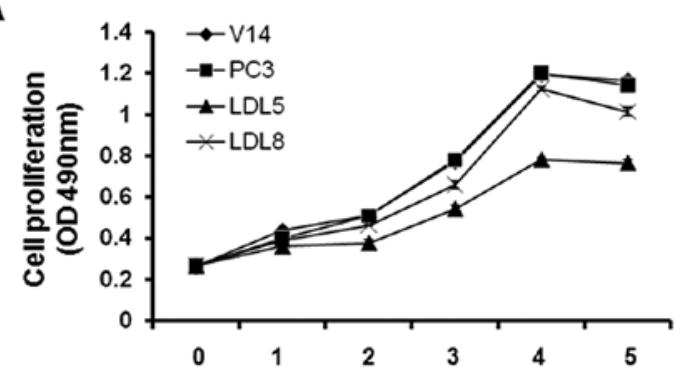

B

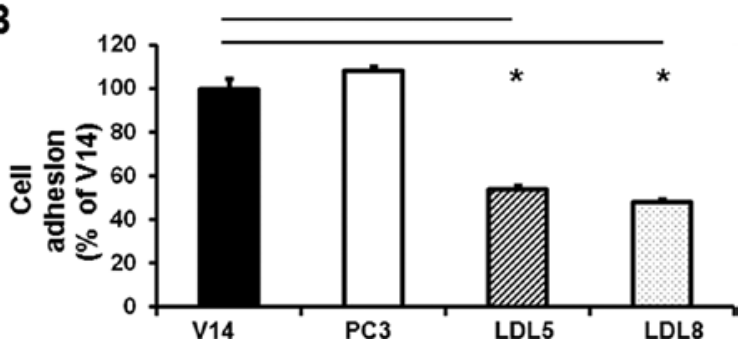

Figure 2. (A) Effect of RXFP1-LDL-A expression on PC3 cell proliferation. No difference was detected between non-transfected parental PC 3 cells and vector control cells (V14). Cell proliferation was measured daily for 5 days and presented as an absorbance at $490 \mathrm{~nm}$. Two RXFP1-LDL-A transfected cell lines have reduced proliferation. The difference between LDL5 and V14 becomes significant after $24 \mathrm{~h}$ and between LDL8 and V14 after $48 \mathrm{~h}$. All measurements were done in quadruplicate, and the experiments were repeated three times. Data are shown as means \pm SEM. (B) The RXFP1LDL-A expression inhibits PC3 cell adhesion to type I collagen. Cells were seeded into a plate precoated with type I collagen, and incubated for $1 \mathrm{~h}$. Results are normalized to V14 cells and expressed as means \pm SEM. ${ }^{*} \mathrm{P}<0.001$ vs. V14 cells.

of biological responses including cell growth, proliferation and survival (20). To determine whether RXFP1-LDL-A expression impact Akt activation, we assessed the levels of phospho-Akt in LDL5 and V14 cells as well as in PC3 cells transfected with siRNA targeting RXFP1 expression. The data in Fig. 4C shows that the expression of RXFP1-LDL-A was able to inhibit the Akt phosphorylation. The same effect was observed in PC3 cells with a knock-down of RXFP1 expression by siRNA (Fig. 4D).

The QRT-PCR with RNA isolated from control (V14) and RXFP1-LDL-A transfected (LDL5) cell lines was performed for several genes previously identified as the potential targets $(17,21,22)$ of relaxin signaling (Fig. 5). All tested genes showed a significant down-regulation.

RXFPI-LDL-A expression suppresses PC3 tumor growth in xenografts. Since RXFP1-LDL-A expression inhibited several tumorigenic properties of the cancer cells in vitro, we investigated whether RXFP1-LDL-A would alter the growth of the xenografts derived from PC3 cells in nude mice. The LDL5 and V14 cells were inoculated subcutaneously both into the left and right flanks of the intact 2-month old nude males. The tumor take in the groups did not differ [10/12 (83.33\%) in both groups]. The tumor growth was monitored for the next eight weeks. The LDL5 tumors grew slower than the control V14 tumors $(\mathrm{P}<0.001$, two-way ANOVA). At day 36 post inoculation the difference in the tumor volume became statistically significant (Fig. 6A). At the end of the experiment the difference between two groups remained, however, the average tumor volumes became somewhat closer. As shown in Fig. 6B, the primary tumor weight was decreased 66\% in LDL5 group comparing to control, but the difference was not statistically significant $(\mathrm{P}=0.1387$, Student's t-test). It is possible that a long incubation period led to a gradual loss of transfected protein expression in LDL5 cells.

Histopathological examinations of the tumors revealed no apparent differences among two groups of mice studied. Metastases were not detected in the organs studied except one case of lymph node tumor growth in V14 group. IHC with Ki67 marker was performed to evaluate tumor cell proliferation in the RXFP1-LDL-A expressing LDL5 and vector
A
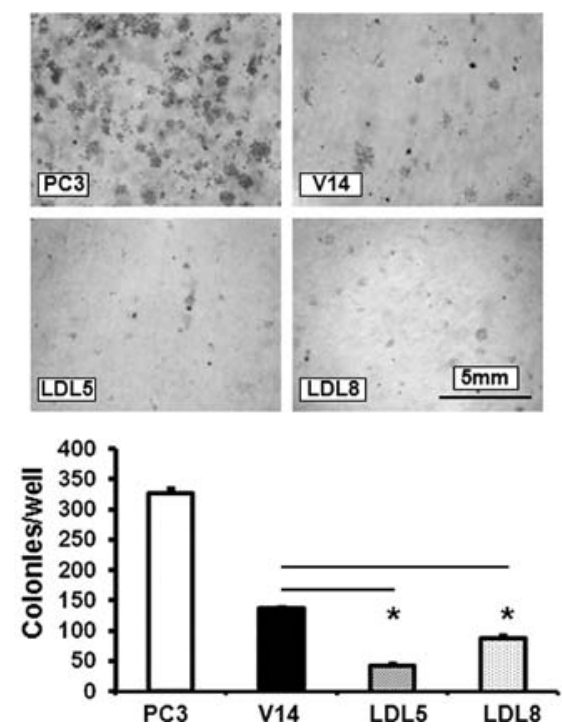

B
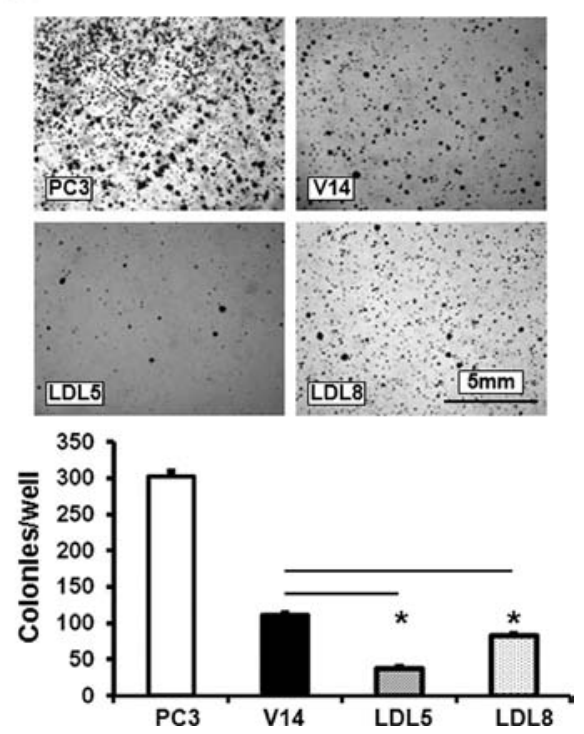

Figure 3. (A) The RXFP1-LDL-A expression suppresses PC3 cell colony formation on a hard surface. The non-transfected cells (PC3) had the highest number of colonies, whereas the RXFP1-LDL-A expressed cells (LDL5 and LDL8) had the lowest number of colonies. (B) The RXFP1-LDL-A expression suppresses PC 3 cell anchorage-independent growth on soft agar. Results are expressed as means \pm SEM. "P $<0.001$ vs. V14 cells. 
A

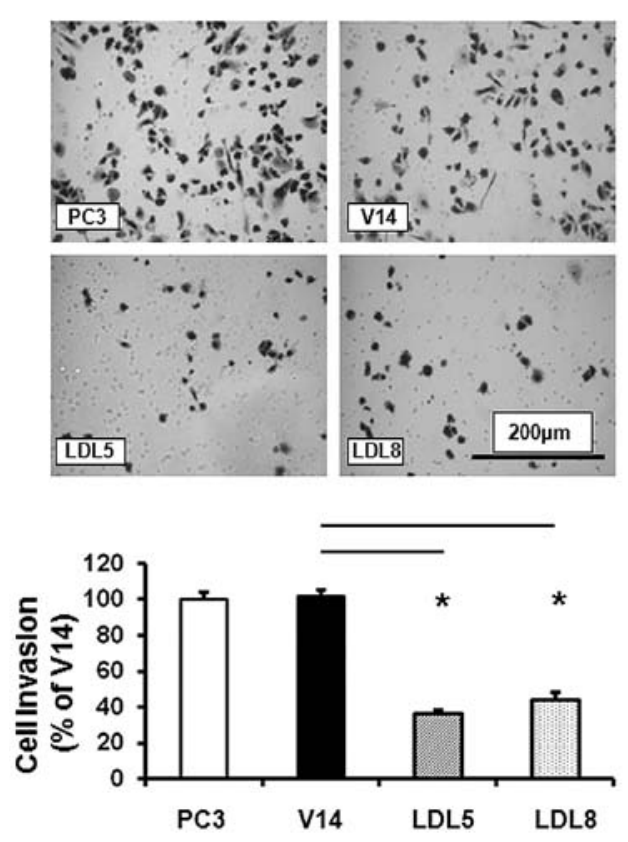

B

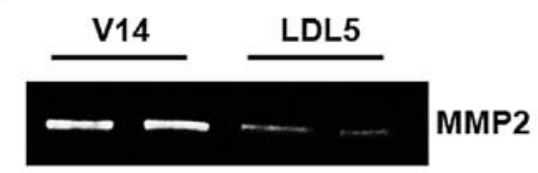

C

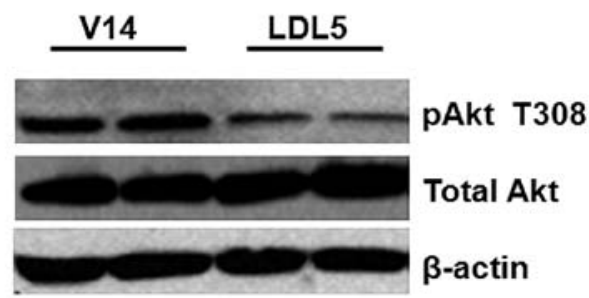

D

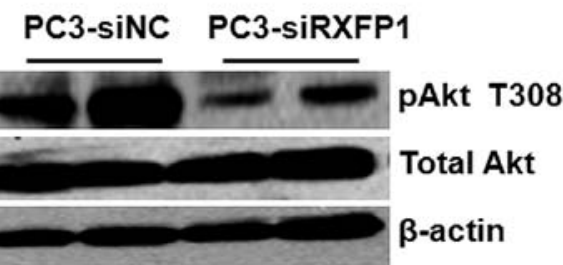

Figure 4. (A) Expression of RXFP1-LDL-A inhibits PC3 cell invasiveness. Experiments were performed with Matrigel invasion chambers. Parental PC3 and vector control V14 showed the same invasion ability. LDL5 and LDL8 stable transfected cells showed reduced invasion, ${ }^{*} \mathrm{P}<0.001$, vs. V14. Cell invasion shown as percentage of V14 control. Results are expressed as means \pm SEM. (B) Expression of RXFP1-LDL-A in PC3 cells inhibited MMP2 activity. MMP-2 activity was evaluated in conditioned media by zymography. (C) Expression of RXFP1-LDL-A or (D) suppression of RXFP1 with specific siRNA (siRXFP1) inhibits Akt phosphorylation in PC3 cells. Cell lysates were analyzed by Western blot hybridization using antibodies to phosphor-Akt (Thr308), total Akt, and $\beta$-actin as internal loading controls. In both siRXFP1 transfected cells and in LDL5 cells the phosphorylation of Akt is suppressed. siNC is a negative control siRNA.

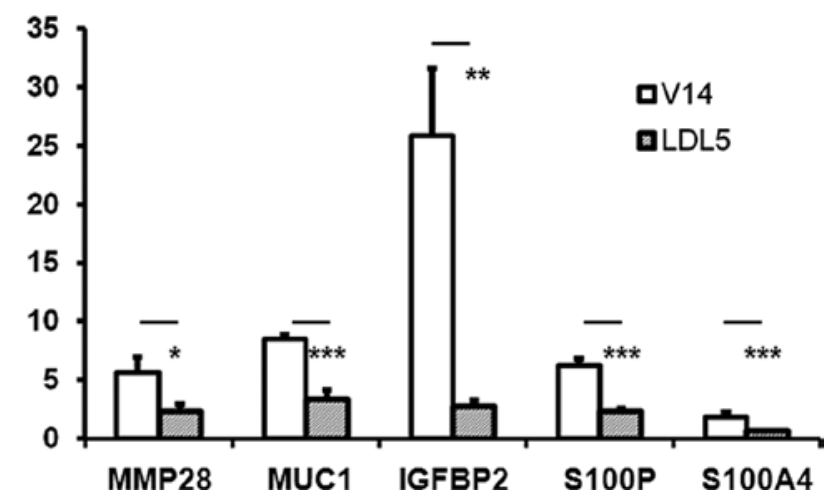

Figure 5. Representative genes down-regulated in PC3 RXFP1-LDL-A stable transfected cells identified by QRT-PCR. Columns represent means; bars are SEM. Differences in gene expression are significant. P-values are calculated by Student's t-test for LDL5 and vector control V14 cells. ${ }^{*} \mathrm{P}<0.05 ;{ }^{* *} \mathrm{P}<0.01$; ${ }^{* * * *} \mathrm{P}<0.001$.

control V14 tumors. The lower expression level of Ki67 in LDL5 xenografts compared to the vector control V14 group was statistically significant (Fig. 6C).

\section{Discussion}

The role of relaxin has been recently demonstrated in breast, endometrial, thyroid, and prostate cancer progression (23). As other growth factors expressed in cancer cells relaxin acts as an autocrine/paracrine stimulus increasing proliferation, invasiveness, and cell adhesion. Additionally, it was shown that relaxin increases vascularization and angiogenesis either directly or indirectly through the stimulation of VEGF or other cell signals. An increased relaxin expression in cancer samples was associated with the more aggressive disease and a decline in survival of the patients $(13,24)$. Several attempts have been made to evaluate the effects of relaxin and relaxin receptor suppression as a potential therapeutic strategy. Such experiments included the analysis of tumor growth using prostate cancer cells stably transfected with relaxin peptide homolog with antagonistic properties (14) and nanoparticle delivery of siRNA targeting RXFP1 (17). Here, we used prostate cancer PC3 cells overexpressing LDL-A module of the relaxin receptor. We have determined that an overexpression of this peptide suppressed growth, adhesion, colony formation, invasiveness of prostate cells in vitro, and xenograft tumor growth in vivo.

The RXFP1 receptor belongs to the leucine rich repeat (LRR) G protein-coupled receptor family (2). The unique feature of this receptor and the related RXFP2 receptor of insulin-like 3 peptide is the presence of $\mathrm{N}$-terminus low-density lipoprotein receptor type A module. Using various mutant and chimeric receptors it was shown that the binding of relaxin by LRR is not sufficient for activation of the receptor (18). No cAMP activation occurred in receptors lacking LDL-A or in mutants with distorted structure of this module (18). As a result, a three step model of relaxin receptor activation was proposed involving relaxin binding by LRR, its interaction with the extracellular loops, and the LDL-A directed receptor activation (19). Significantly, the soluble RXFP1-LDL-A peptide was able to suppress cAMP activation in cells with 
A

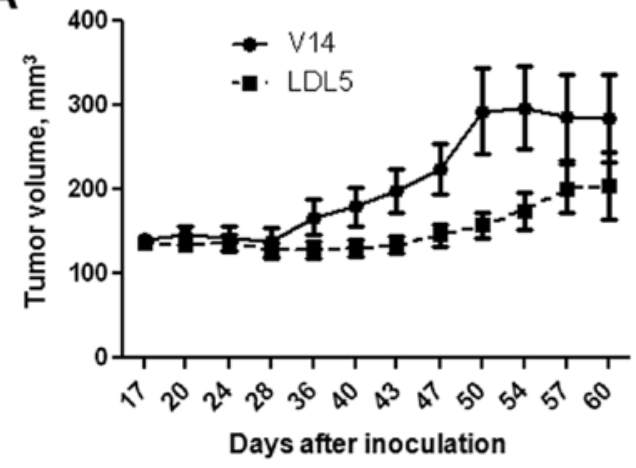

B

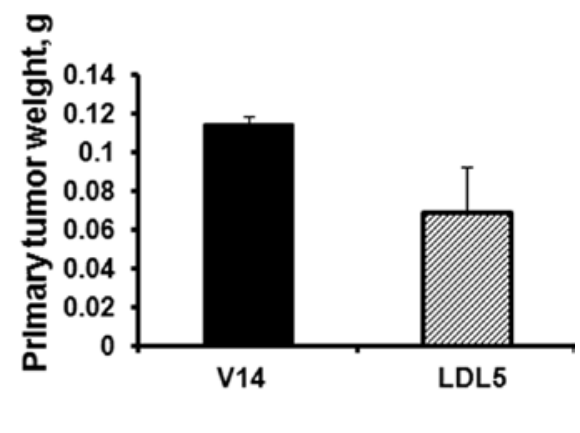

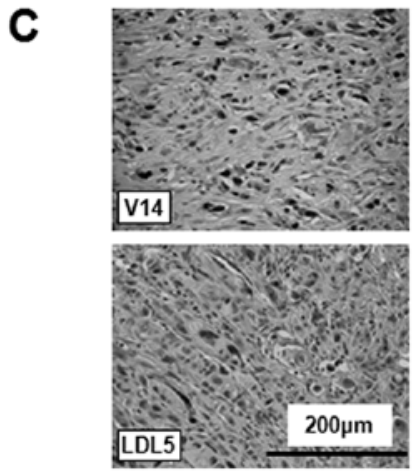

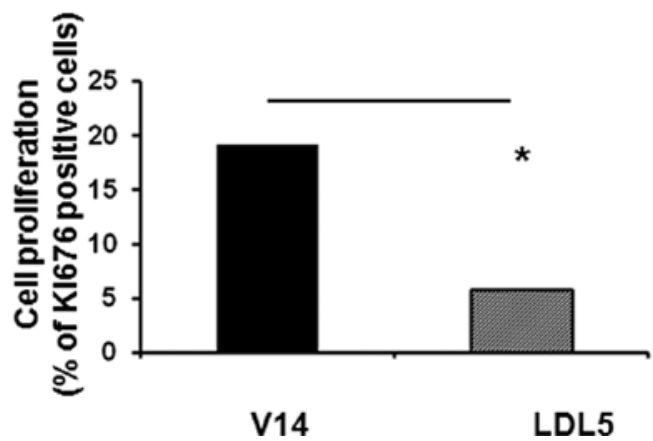

Figure 6. The RXFP1-LDL-A expression suppresses PC3 cell xenograft growth in nude mice. (A) Growth of LDL5 and V14 cells xenografts in nude mice was monitored for 60 days after cancer cell injections in both flanks by measuring tumor volume twice a week. Results are expressed as means \pm SEM. The growth of LDL5 xenografts was slower than V14 xenografts (two-way ANOVA, P<0.001). Mean tumor volumes in LDL5 and V14 were significantly different from day 24 to 50 (Student's t-test, $\mathrm{P}<0.05$ ). (B) The tumor weight at the end of the 2 months. Results are expressed as means \pm SEM. (C) Decreased expression of proliferation marker Ki67 in LDL5 vs PC3-V14 xenografts. Representative photomicrographs show IHC staining for Ki67 (black nuclei) in tumor sections of LDL5 and V14 xenografts. Results are expressed as means \pm SEM; ${ }^{*} \mathrm{P}<0.001$.

transfected RXFP1 (4). Injections of the recombinant peptide corresponding to the whole extracellular domain of RXFP1 had a suppressive effect on nipple development and delayed parturition in mice (25). Multiple splice variants of RXFP1 gene, some of them containing LDL-A module have been detected in numerous tissues $(19,26,27)$. It was suggested that expression of such peptides may be responsible for a modulation of relaxin signaling.

The PC3 cells express relaxin receptor RXFP1, however, the stimulation with hormone does not cause an appreciable increase in cAMP as in receptor transfected HEK293 cells or in monocytic leukemia THP1 cells. Thus, we evaluated the effect of LDL-A transfection on the known responses of these cells to relaxin stimulation. It has been shown previously that the addition of relaxin could cause an increase in invasiveness, migration, cell adhesion, colony formation, as well as an increase in cell proliferation (10-13,15-17). Suppression of relaxin signaling using siRNA targeting hormone or receptor mRNAs had an opposite effect $(13,17)$. We showed here that the transfection with RXFP1- LDL-A indeed affected all of these cellular functions in vitro, and significantly suppressed tumor growth in vivo.

Several potential cell signaling mechanisms controlled by relaxin have been described. In androgen receptor positive LNCaP cells relaxin activates AR through an activation of $\beta$-catenin, which is accompanied by phosphatidylinositol 3-kinase (PI3K)/protein kinase B (Akt) activation and phosphorylation/degradation of glycogen synthase kinase-3 $\beta$
(GSK-3 $\beta$ ) (15). An activation of $\mathrm{Wnt} / \beta$-catenin pathway has been also demonstrated in LNCaP cells overexpressing relaxin, through the activation of Protocadherin Y (PCDHY) and stabilization/nuclear translocation of $\beta$-catenin (16). Both relaxin and PCDHY are negatively regulated by androgens $(12,16)$. We demonstrated here that the siRNA suppression of RXFPl and the LDL-A expression in PC3 cells resulted in significant decrease of Akt phosphorylation, suggesting a common relaxin/ $\beta$-catenin/Akt activation pathway in both PC3 and $\mathrm{LNCaP}$ prostate cell lines.

Previously we have performed a whole genome expression analysis of PC3 cells transfected with siRNA against RXFP1 (17). Remarkably, several missregulated genes were common between two experiments. In current experiment, the LDL-A was expressed in a stable manner, and thus, had a long-term effect on cancer cells. In siRNA knockdown treatment the effect of an acute down-regulation of relaxin signaling in PC3 cells was evaluated. The well-established modulators of prostate cancer progression such as MUC1, IGFBP2, S100A4, previously identified as the targets of relaxin signaling were also missregulated in RXFP1-LDL-A expressing PC3 cells $(17,28)$. Although we cannot exclude some non-specific effects of RXFP1-LDL-A stable expression, the data indicate that relaxin signaling might be affected in transfected cells.

In summary, we have shown that the overexpression of RXFP1-LDL-A domain in prostate adenocarcinoma PC3 cells reduced their tumorigenic properties and thus identify 
relaxin signaling as a potential therapeutic target for cancer treatment.

\section{Acknowledgements}

The authors thank Mrs. Anne Truong for excellent technical assistance. We thank Dr Sheau Yu Teddy Hsu, Stanford University School of Medicine for a gift of RXFP1 plasmid. This study was supported by the National Cancer Institute grant R21CA118362 to A.I.A.

\section{References}

1. Sherwood OD: Relaxin's physiological roles and other diverse actions. Endocr Rev 25: 205-234, 2004.

2. Bathgate RA, Ivell R, Sanborn BM, Sherwood OD and Summers RJ: International Union of Pharmacology LVII recommendations for the nomenclature of receptors for relaxin family peptides. Pharmacol Rev 58: 7-31, 2006.

3. Agoulnik AI: Relaxin and related peptides in male reproduction. Adv Exp Med Biol 612: 49-64, 2007.

4. Hsu SY, Nakabayashi K, Nishi S, et al: Activation of orphan receptors by the hormone relaxin. Science 295: 671-674, 2002.

5. Zhao L, Roche PJ, Gunnersen JM, et al: Mice without a functional relaxin gene are unable to deliver milk to their pups. Endocrinology 140: 445-453, 1999.

6. Krajnc-Franken MA, van Disseldorp AJ, Koenders JE, Mosselman S, van Duin M and Gossen JA: Impaired nipple development and parturition in LGR7 knockout mice. Mol Cel Biol 24: 687-696, 2004.

7. Kamat AA, Feng S, Bogatcheva NV, Truong A, Bishop CE and Agoulnik AI: Genetic targeting of relaxin and insulin-like factor 3 receptors in mice. Endocrinology 145: 4712-4720, 2004.

8. Samuel CS, Hewitson TD, Unemori EN and Tang ML: Drugs of the future: the hormone relaxin. Cell Mol Life Sci 64: 1539-1557, 2007.

9. Jeyabalan A, Shroff SG, Novak J and Conrad KP: The vascular actions of relaxin. Adv Exp Med Biol 612: 65-87, 2007.

10. Vinall RL, Tepper CG, Shi XB, Xue LA, Gandour-Edwards R and de Vere White RW: The R273H p53 mutation can facilitate the androgen-independent growth of LNCaP by a mechanism that involves $\mathrm{H} 2$ relaxin and its cognate receptor LGR7. Oncogene 25: 2082-2093, 2006

11. Silvertown JD, Ng J, Sato T, Summerlee AJ and Medin JA: H2 relaxin overexpression increases in vivo prostate xenograft tumor growth and angiogenesis. Int J Cancer 118: 62-73, 2006.

12. Thompson VC, Morris TG, Cochrane DR, et al: Relaxin becomes upregulated during prostate cancer progression to androgen independence and is negatively regulated by androgens. Prostate 66: 1698-1709, 2006.
13. Feng S, Agoulnik IU, Bogatcheva NV, et al: Relaxin promotes prostate cancer progression. Clin Cancer Res 13: 1695-1702, 2007.

14. Silvertown JD, Symes JC, Neschadim A, et al: Analog of H2 relaxin exhibits antagonistic properties and impairs prostate tumor growth. FASEB J 21: 754-765, 2007.

15. Liu S, Vinall RL, Tepper C, et al: Inappropriate activation of androgen receptor by relaxin via beta-catenin pathway. Oncogene 27: 499-505, 2008

16. Thompson VC, Hurtado-Coll A, Turbin D, et al: Relaxin drives Wnt signaling through upregulation of PCDHY in prostate cancer. Prostate 70: 1134-1145, 2010.

17. Feng S, Agoulnik IU, Truong A, et al: Suppression of relaxin receptor RXFP1 decreases prostate cancer growth and metastasis. Endocr Relat Cancer 17: 1021-1033, 2010.

18. Kong RC, Shilling PJ, Lobb DK, Gooley PR and Bathgate RA: Membrane receptors: structure and function of the relaxin family peptide receptors. Mol Cell Endocrinol 320: 1-15, 2010.

19. Scott DJ, Layfield S, Yan Y, et al: Characterization of novel splice variants of LGR7 and LGR8 reveals that receptor signaling is mediated by their unique low density lipoprotein class A modules. J Biol Chem 281: 34942-34954, 2006.

20. de Souza PL, Russell PJ and Kearsley J: Role of the Akt pathway in prostate cancer. Curr Cancer Drug Targets 9: 163-175, 2009.

21. Radestock Y, Willing C, Kehlen A, Hoang-Vu C and HombachKlonisch S: Relaxin enhances S100A4 and promotes growth of human thyroid carcinoma cell xenografts. Mol Cancer Res 8: 494-506, 2010.

22. Ohleth KM, Lenhart JA, Ryan PL, Radecki SV and Bagnell CA: Relaxin increases insulin-like growth factors (IGFs) and IGF-binding proteins of the pig uterus in vivo. Endocrinology 138: 3652-3658, 1997.

23. Klonisch T, Bialek J, Radestock Y, Hoang-Vu C and HombachKlonisch S: Relaxin-like ligand-receptor systems are autocrine/ paracrine effectors in tumor cells and modulate cancer progression and tissue invasiveness. Adv Exp Med Biol 612: 104-118, 2007.

24. Kamat AA, Feng S, Agoulnik IU, et al: The role of relaxin in endometrial cancer. Cancer Biol Ther 5: 71-77, 2006.

25. Hsu SY, Nakabayashi K, Nishi S, et al: Relaxin signaling in reproductive tissues. Mol Cell Endocrinol 202: 165-170, 2003.

26. Kern A, Agoulnik AI and Bryant-Greenwood GD: The lowdensity lipoprotein class A module of the relaxin receptor (leucine-rich repeat containing G-protein coupled receptor 7): its role in signaling and trafficking to the cell membrane. Endocrinology 148: 1181-1194, 2007.

27. Kern A, Hubbard D, Amano A and Bryant-Greenwood GD Cloning, expression, and functional characterization of relaxin receptor (leucine-rich repeat-containing $G$ protein-coupled receptor 7) splice variants from human fetal membranes. Endocrinology 149: 1277-1294, 2008.

28. Radestock Y, Hoang-Vu C and Hombach-Klonisch S: Relaxin downregulates the calcium binding protein S100A4 in MDA-MB231 human breast cancer cells. Ann N Y Acad Sci 1041: 462-469, 2005. 